(C) The Authors 2019. This is an Open Access article, distributed under the terms of the Creative Commons Attribution licence (http:// creativecommons.org/licenses/by/4.0/), which permits unrestricted re-use, distribution, and reproduction in any medium, provided the original work is properly cited.

\title{
Sex difference of the predictive value of BMI, waist circumference and percentage body fat mass for gallstone disease
}

\author{
Hsin-Yin $\mathrm{Hsu}^{1}$, Chun-Yuan Huang ${ }^{2}$ and Lee-Ching Hwang ${ }^{1,3 *}$ \\ ${ }^{1}$ Department of Family Medicine, MacKay Memorial Hospital, Taipei, 10449, Taiwan \\ ${ }^{2}$ Department of Family Medicine, Chinese Medical University Hospital Taipei Branch, Taipei, 11449, Taiwan \\ ${ }^{3}$ Department of Medicine, Mackay Medical College, New Taipei City, 25245, Taiwan
}

(Submitted 14 August 2018 - Final revision received 6 January 2019 - Accepted 4 February 2019 - First published online 11 February 2019)

\section{Abstract}

Gallstone disease (GSD) represents a significant health burden and is reported to be strongly related to obesity. This study examined the effect of sex on the association of BMI, waist circumference (WC) and percentage body fat mass (\%FM) with GSD. We enrolled 15671 middle-aged Taiwanese adults undergoing a physical check-up at a health examination centre. Multiple logistic regression analysis was conducted to identify the association of BMI, WC and \%FM with the presence of GSD. AUC of the receiver-operating characteristic (ROC) curves were calculated for BMI, WC and \%FM to compare their respective ability to correctly classify patients with GSD. From our findings, GSD prevalence was $8.1 \%$ in men and $6.3 \%$ in women. The percentage of markedly increased obesity indicators was significantly higher in patients with GSD than in those without GSD. Multivariate analysis showed that all obesity indicators were significant predictors of the presence of GSD in women after adjustment. In men, only BMI and WC were significantly associated with the presence of GSD. In the ROC curve analysis, BMI and WC were the best predictors of GSD risk in women and men, respectively. The obesity indicators better predicted the presence of GSD in women than in men. In conclusion, the best obesity indicator of GSD risk differed by sex. In addition to body weight, fat mass and central obesity were associated with GSD risk in women. In men, central obesity may play a more important role in predicting GSD risk.

\section{Key words: BMI: Waist circumference measurement: Body fat mass: Gallbladder stone disease}

Gallstone disease (GSD) is the most common biliary tract disease and its prevalence varies from 5 to $25 \%$ worldwide. Although over two thirds of patients with gallstones remain asymptomatic, GSD represents a significant medical burden because of the associated risks of cholecystitis, pancreatitis, gallbladder cancer and the development of major cardiometabolic diseases ${ }^{(1-3)}$.

Epidemiological studies report that advanced age, the female sex, race, oral contraceptive use, diabetes mellitus, family history and obesity are commonly reported risk factors for GSD development ${ }^{(3,4)}$. Although the risk factors for GSD vary by geographic area and ethnicity, obesity remains a traditional risk factor for $\mathrm{GSD}^{(5-7)}$. Only a few studies demonstrated the association between obesity and GSD development using different standards to define obesity. Tsai $e t a l .{ }^{(8)}$, in Kentucky, reported that central obesity and regional fat distribution are independent risk factors for GSD-related cholecystectomy in women. Radmard et al. ${ }^{(9)}$ demonstrated that the best anthropometric parameter for GSD risk in men is the waist:hip ratio. Another study reported that multiple obesity indicators are associated with the risk of GSD. However, it is unclear which obesity indicator provides the strongest association with GSD risk. Therefore, we evaluated three obesity indicators in the current study: BMI as an indicator for general obesity, waist circumference (WC) for the estimation of central obesity, and percentage body fat mass (\%FM) to evaluate general fat mass. We examined the relationship of BMI, WC and \%FM with GSD, aiming to determine which obesity index has the strongest association with the risk of GSD in men and women.

\section{Methods}

\section{Study population}

A total of 15671 middle-aged Taiwanese adults undergoing a physical check-up at a Northern Taiwan health examination centre were recruited for the study. We excluded those with viral hepatitis, heavy alcohol consumption, age more than 65 years, those with a history of cholecystectomy or those who did not undergo abdominal ultrasonographic examination or fasting blood testing. This study was conducted according to the guidelines laid down in the Declaration of Helsinki, and all procedures involving human subjects were approved by Mackay Memorial Hospital, Taipei, Taiwan (approval number

Abbreviations: \%FM, percentage body fat mass; GSD, gallstone disease; WC, waist circumference.

* Corresponding author: L.-C. Hwang, fax +886 2 25213847, email hlc@mmh.org.tw 
12MMHIS092). Verbal consent was witnessed and formally recorded.

\section{Study methods}

This was a cross-sectional study. The data gathered included age, sex, height, weight, obesity indicators (BMI, WC and \%FM measured by bioelectric impedance analysis: Tanita, BC-418), exercise habit (regular exercise defined as $>3$ times/week) and history of hypertension or diabetes mellitus (defined as ever being diagnosed with hypertension or diabetes mellitus, regardless of the prescription of medication). We also collected standard biochemical data including fasting blood glucose, lipid profile and renal and liver function tests. According to previous studies, the positive and negative predictive values of abdominal sonography for GSD are approximately $99-100$ and $90-96 \%{ }^{(10)}$, respectively. GSD was diagnosed according to the presence of gallstones on abdominal sonography after fasting for at least $8 \mathrm{~h}$. Obesity was defined as BMI $\geq 27 \mathrm{~kg} / \mathrm{m}^{2}$, WC $\geq 80 \mathrm{~cm}$ in women and $\geq 90 \mathrm{~cm}$ in men or body fat $\geq 7 \%$ in women and $\geq 23 \%$ in men, using the criteria defined by the Health Promotion Administration, Ministry of Health and Welfare in Taiwan.

\section{Statistical analyses}

SPSS for Windows version 24.0 (IBM Corp.) and R Studio version 1.1.45 were used for all statistical analyses. Continuous variables, such as age, BMI, WC and \%FM, are expressed as means and standard deviations. Categorical variables included obesity (by each obesity standard), exercise and history of hypertension and diabetes mellitus. The $\chi^{2}$ test was used to analyse the difference in the GSD rate among each of these categorical variables. Univariate analyses were used to select variables that predicted the risk of GSD. All variables that predicted the risk of GSD in the univariate analyses were entered into a multiple logistic regression analysis, controlling for potential confounders, including age and sex. Multiple logistic regression analysis was conducted to identify the association between BMI, WC and \%FM and the presence of GSD. AUC of the receiver-operating characteristic (ROC) curves were calculated for BMI, WC and \%FM to compare their relative ability to correctly identify patients with GSD using the methodology of DeLong et $a l^{(11)}$. Results are presented as OR with 95\% CI. Statistical significance was set at $P<0.05$.

\section{Results}

The 15671 included participants were stratified by sex (men: 8146, 52.0\% and women: $7525,48.0 \%$ ) (Table 1). The male group was older than the female group ( $43.1 v$. $40 \cdot 8$ years, $P<0.001)$ and had a significantly higher rate of comorbid hypertension or diabetes mellitus. Higher biochemical parameter levels including fasting plasma glucose, total cholesterol level and TAG were found in the male group. Obesity parameters (BMI, WC and \%FM) were all significantly higher in men than in women. Men had a significantly higher rate of a regular exercise habit. Men also had a significantly higher rate of GSD than women $(8.3 v \cdot 6.3 \%, P<0.001)$.
Table 2 compares parameters according to the presence or absence of GSD between men and women. Among men, 675 patients $(8 \cdot 3 \%)$ had GSD; men with GSD were older than the men without GSD (48.9v $v .42 .5$ years, $P<0.001$ ). Men with GSD had a higher rate of obesity $(29 \cdot 1 v .23 \cdot 6 \%, P<0 \cdot 001)$ based on BMI $\geq 27 \mathrm{~kg} / \mathrm{m}^{2}$ and the presence of central obesity ( 45.5 v. $35.5 \%, P<0.001$ ) based on $\mathrm{WC} \geq 90 \mathrm{~cm}$. However, there was no statistical difference in the rate of a higher $\% \mathrm{FM}$ $(\geq 23 \%)$ between men with and without GSD (62.1 v. 58.7\%, $P<0 \cdot 100)$. The rate of regular exercise was similar between men with and without GSD. Men with GSD had a higher rate of comorbid hypertension $(31.3 v .19 \cdot 6 \%, P<0.001)$ and diabetes mellitus $(12.9 v \cdot 6.9 \%, P<0.001)$ than men without GSD. In the female group, 473 patients (6.3\%) had GSD; women with GSD were older than women without GSD $(46 \cdot 1 v$. 40.4 years, $P<0.001)$. The three obesity parameters were all significantly higher in women with GSD than in women without GSD (BMI $\geq 27 \mathrm{~kg} / \mathrm{m}^{2}, 16 \cdot 1 v \cdot 7 \cdot 6 \%, P<0 \cdot 001$; WC $\geq 80 \mathrm{~cm}, 45 \cdot 5 v$. $23.9 \%, P<0.001 ; \% \mathrm{FM} \geq 7 \%, 74.8 v .54 .6 \%, P<0.001)$. The rate of regular exercise was similar between women with and without GSD. Women with GSD had a higher rate of hypertension $(15.9 v .7 .5 \%, P<0.001)$ and diabetes mellitus $(7.6 v$. $3.2 \%, P<0.001)$ compared to women without GSD.

Multiple logistic regression was performed to analyse the association between obesity parameters and GSD by sex (Table 3); the OR was adjusted by age and the presence of hypertension and diabetes mellitus. In the male group, \%FM had no statistically significant association with GSD. Independent predictors of GSD in men were BMI $(\mathrm{OR}=1 \cdot 33, P=0.003)$, central obesity $(\mathrm{OR}=1 \cdot 26, P=0.007)$ and the combination of the three obesity parameters $(\mathrm{OR}=1.47, P<0.001)$. In the female group, the independent predictors of GSD were $\% \mathrm{FM}$ (OR=1.94, $P<0.001)$, BMI $(\mathrm{OR}=1.83, \quad P<0.001)$, central obesity (OR $=2 \cdot 00, P<0.001)$ and the combination of the three obesity parameters (OR $=2 \cdot 76, P<0 \cdot 001)$ (Fig. 1).

Table 4 shows the ROC analysis for the association of BMI, WC and \%FM with GSD. The results showed that, in men, the AUC for WC was largest (AUC: 0-683). For women, the AUC was largest for BMI (AUC: 0.693). The AUC for each obesity indicator was slightly larger in women than in men.

\section{Discussion}

Our study revealed that the use of different obesity indicators leads to a variable distribution of obesity among men and women in Taiwan. We found that, compared with central obesity and BMI, obesity defined as a higher \%FM resulted in the largest proportion of patients diagnosed with obesity in both men and women. The discrepancy in the percentage of participants described as obese between these obesity indicators might be because BMI focuses only on the ratio between weight and square of height and ignores the importance of adiposity; thus, potentially leading to misclassification of non-obese individuals who do not have a high \%FM or regional obesity. In previous studies, Peltz et al. ${ }^{(12)}$ reported that \%FM could more accurately assess obesity in Mexican Americans compared with BMI. Considering the differences in obesity diagnosis between 
Table 1. Demographic and basic characteristics of the study population* (Mean values and standard deviations; numbers and percentages)

\begin{tabular}{|c|c|c|c|c|c|}
\hline & \multicolumn{2}{|c|}{ Men } & \multicolumn{2}{|c|}{ Women } & \multirow[b]{3}{*}{$P$} \\
\hline & \multicolumn{2}{|c|}{$n 8146(52 \cdot 0 \%)$} & \multicolumn{2}{|c|}{$n 7525(48.0 \%)$} & \\
\hline & Mean & SD & Mean & SD & \\
\hline Age (years) & $43 \cdot 1$ & 10.5 & $40 \cdot 8$ & $10 \cdot 7$ & $<0.001$ \\
\hline Fasting plasma glucose $(\mathrm{mmol} / \mathrm{l})$ & 5.7 & 1.0 & 5.4 & 0.8 & $<0.001$ \\
\hline Total cholesterol level (mmol/l) & $5 \cdot 1$ & 0.9 & 4.9 & 0.9 & $<0.001$ \\
\hline TAG $(\mathrm{mmol} / \mathrm{l})$ & 1.6 & $1 \cdot 2$ & 1.0 & 0.6 & $<0.001$ \\
\hline BMI $\left(\mathrm{kg} / \mathrm{m}^{2}\right)$ & $25 \cdot 0$ & 3.4 & $22 \cdot 0$ & 3.5 & $<0.001$ \\
\hline General obesity $\dagger$ & & & & & $<0.001$ \\
\hline$n$ & & & & & \\
\hline$\%$ & & & & & \\
\hline Waist circumference $(\mathrm{cm})$ & $87 \cdot 2$ & $9 \cdot 2$ & $75 \cdot 1$ & $8 \cdot 6$ & $<0.001$ \\
\hline Central obesity $\ddagger$ & & & & & $<0.001$ \\
\hline$n$ & & & & & \\
\hline$\%$ & & & & & \\
\hline Body fat $(\%)$ & 24.9 & $5 \cdot 6$ & $29 \cdot 0$ & $6 \cdot 8$ & $<0.001$ \\
\hline High percentage body fat mass $\S$ & & & & & $<0.001$ \\
\hline$n$ & & & & & \\
\hline$\%$ & & & & & \\
\hline Gallstone & & & & & $<0.001$ \\
\hline$n$ & & & & & \\
\hline$\%$ & & & & & \\
\hline Exercise & & & & & $<0.001$ \\
\hline$n$ & & & & & \\
\hline$\%$ & & & & & \\
\hline Hypertension & & & & & $<0.001$ \\
\hline$n$ & & & & & \\
\hline$\%$ & & & & & \\
\hline Diabetes mellitus & & & & & $<0.001$ \\
\hline$n$ & & & & & \\
\hline$\%$ & & & & & \\
\hline
\end{tabular}

* Continuous variables are shown as means and standard deviations; categorical variables are shown as numbers and percentages. † General obesity indicated by BMI $\geq 27 \mathrm{~kg} / \mathrm{m}^{2}$.

¥ Central obesity indicated by waist circumference $\geq 80 \mathrm{~cm}$ in women and $\geq 90 \mathrm{~cm}$ in men.

$\S$ Higher percentage body fat mass indicated by body fat $\geq 27 \%$ in women and $\geq 23 \%$ in men.

Table 2. Demographic and obesity parameters according to the presence of gallstone disease by sex (Numbers and percentages; mean values and standard deviations)

\begin{tabular}{|c|c|c|c|c|c|c|c|c|c|c|}
\hline & \multicolumn{5}{|c|}{ Men } & \multicolumn{5}{|c|}{ Women } \\
\hline & \multicolumn{2}{|c|}{ No gallstones } & \multicolumn{2}{|c|}{ Gallstone } & \multirow[b]{2}{*}{$P$} & \multicolumn{2}{|c|}{ No gallstones } & \multicolumn{2}{|c|}{ Gallstone } & \multirow[b]{2}{*}{$P$} \\
\hline & $n$ & $\%$ & $n$ & $\%$ & & $n$ & $\%$ & $n$ & $\%$ & \\
\hline$n$ & 7471 & $91 \cdot 7$ & 675 & $8 \cdot 3$ & & 7052 & 93.7 & 473 & $6 \cdot 3$ & \\
\hline Age (years) & & & & & $<0.001$ & & & & & $<0.001$ \\
\hline Mean & \multicolumn{2}{|c|}{$42 \cdot 5$} & \multicolumn{2}{|c|}{$48 \cdot 9$} & & \multicolumn{2}{|c|}{$40 \cdot 4$} & \multicolumn{2}{|c|}{$46 \cdot 1$} & \\
\hline $\mathrm{SD}$ & \multicolumn{2}{|c|}{10.4} & \multicolumn{2}{|c|}{$10 \cdot 0$} & & \multicolumn{2}{|c|}{$10 \cdot 6$} & \multicolumn{2}{|c|}{10.5} & \\
\hline General obesity* & 1763 & $23 \cdot 6$ & 196 & $29 \cdot 1$ & $<0.001$ & 535 & $7 \cdot 6$ & 76 & $16 \cdot 1$ & $<0.001$ \\
\hline Central obesity $†$ & 2653 & $35 \cdot 5$ & 307 & $45 \cdot 5$ & $<0.001$ & 1687 & 23.9 & 215 & $45 \cdot 5$ & $<0.001$ \\
\hline Higher percentage body fat mass $\ddagger$ & 4385 & $58 \cdot 7$ & 419 & $62 \cdot 1$ & 0.100 & 3850 & $54 \cdot 6$ & 354 & 74.8 & $<0.001$ \\
\hline Exercise & 2144 & $28 \cdot 7$ & 201 & $29 \cdot 8$ & 0.553 & 1499 & $21 \cdot 3$ & 104 & $22 \cdot 0$ & 0.707 \\
\hline Hypertension & 1465 & $19 \cdot 6$ & 211 & $31 \cdot 3$ & $<0.001$ & 529 & 7.5 & 75 & $15 \cdot 9$ & $<0.001$ \\
\hline Diabetes mellitus & 512 & 6.9 & 81 & $12 \cdot 0$ & $<0.001$ & 225 & 3.2 & 36 & $7 \cdot 6$ & $<0.001$ \\
\hline
\end{tabular}

* General obesity indicated by $\mathrm{BMI} \geq 27 \mathrm{~kg} / \mathrm{m}^{2}$.

† Central obesity indicated by waist circumference $\geq 80 \mathrm{~cm}$ in women and $\geq 90 \mathrm{~cm}$ in men.

$\ddagger$ Higher percentage body fat mass indicated by body fat $\geq 27 \%$ in women and $\geq 23 \%$ in men.

$\% \mathrm{FM}$ and $\mathrm{WC}$, we found that most patients with central obesity were included among those with a higher \%FM.

The prevalence of GSD varies according to ethnicity and geographic location. Studies have shown that Western countries have the highest prevalence of GSD (10-25\%), followed by
Asian regions; African countries show the lowest prevalence of $\operatorname{GSD}^{(1,2,4,13)}$. Most patients with GSD remain asymptomatic ${ }^{(1,14)}$, potentially leading to an underestimation of the prevalence of GSD, especially among individuals living in areas where routine physical examinations are uncommon. In our studies, the 
Table 3. Multiple logistic regression analysis of the association between obesity parameters and gallstone disease by sex (Odds ratios and $95 \%$ confidence intervals)

\begin{tabular}{|c|c|c|c|c|c|c|}
\hline \multirow[b]{2}{*}{ Variables } & \multicolumn{3}{|c|}{ Men } & \multicolumn{3}{|c|}{ Women } \\
\hline & OR & $95 \% \mathrm{Cl}$ & $P$ & OR & $95 \% \mathrm{Cl}$ & $P$ \\
\hline Higher percentage body fat mass & $1 \cdot 15$ & $0.96,1.36$ & 0.124 & 1.94 & $1.56,2.42$ & $<0.001$ \\
\hline General obesity & 1.33 & $1 \cdot 10,1.60$ & 0.003 & $1 \cdot 83$ & $1 \cdot 38,2 \cdot 42$ & $<0.001$ \\
\hline Central obesity & 1.26 & $1.07,1.49$ & 0.007 & 2.00 & $1.63,2.54$ & $<0.001$ \\
\hline $\begin{array}{l}\mathrm{BMI} \geq 27 \mathrm{~kg} / \mathrm{m}^{2} \text { with central obesity and higher body fat } v \text {. non-obesity } \\
\text { by BMI, body fat and waist circumference }\end{array}$ & 1.47 & $1 \cdot 19,1.82$ & $<0.001$ & $2 \cdot 76$ & $1.99,3.84$ & $<0.001$ \\
\hline
\end{tabular}

* OR was adjusted by age and the presence of hypertension and diabetes.
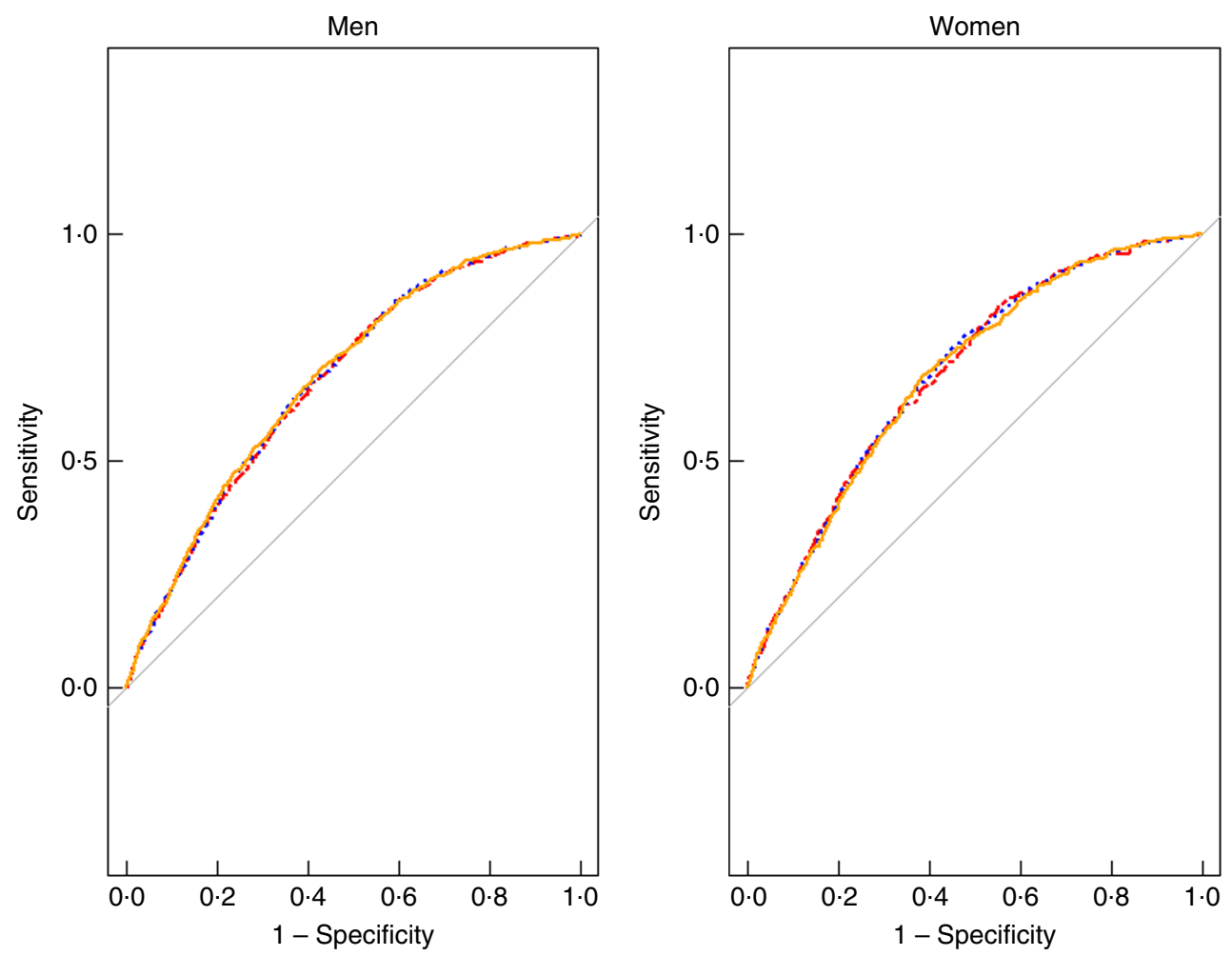

Fig. 1. Receiver-operating characteristic analysis result for the association of BMI (- --_.), waist circumference (WC, $\longrightarrow$ ) and percentage body fat ( - - - .) mass with gallstone disease in both sexes. For men, the AUC for WC was largest (AUC: 0.683). For women, the AUC was largest for BMI (AUC: 0.693).

Table 4. Comparison of the receiver-operating characteristic (ROC) curves of BMI, waist circumference and percentage body fat mass (\%FM) by sex

\begin{tabular}{|c|c|c|c|c|c|c|c|c|}
\hline \multirow[b]{2}{*}{ Variables } & \multicolumn{4}{|c|}{ Men } & \multicolumn{4}{|c|}{ Women } \\
\hline & AUC & $95 \% \mathrm{Cl}$ & Comparison & $P$ & AUC & $95 \% \mathrm{Cl}$ & Comparison & $P$ \\
\hline \multicolumn{9}{|l|}{ ROC } \\
\hline \%FM (\%) & 0.677 & $0.658,0.697$ & BMI & 0.169 & 0.691 & $0.668,0.713$ & BMI & 0.465 \\
\hline BMI $\left(\mathrm{kg} / \mathrm{m}^{2}\right)$ & 0.680 & $0.661,0.700$ & Waist circumference & 0.161 & 0.693 & $0.670,0.715$ & Waist circumference & 0.273 \\
\hline Waist circumference $(\mathrm{cm})$ & 0.683 & $0.663,0.702$ & $\% F M$ & 0.029 & 0.688 & $0.666,0.711$ & $\% F M$ & 0.570 \\
\hline
\end{tabular}

prevalence of GSD among women and men was $8 \cdot 3$ and $6 \cdot 3 \%$, respectively, which is lower than the prevalence reported in other Asian populations ${ }^{(1-3,13)}$. This may be due to the fact that the rate of GSD increases with age ${ }^{(15)}$, while we only enrolled participants aged between 18 and 64 years. In addition, studies in Western countries reveal that the female sex is a traditional risk factor for GSD. The hypothesis that women are prone to
GSD is based on the potential ability of oestrogen to increase the cholesterol concentration in bile, increasing the possibility of cholesterol gallstone formation ${ }^{(10,14)}$. This is consistent with the high prevalence of cholesterol gallstones in the western population. However, this study found that GSD is slightly more prevalent among men than among women. Stringer et al. ${ }^{(16)}$ studied the composition of gallstones according to ethnicity and 
reported that black pigment gallstones (predominantly calcium bilirubinate), often associated with liver disease, bacterial infection and other causes and least commonly associated with obesity, accounted for approximately $40 \%$ of gallstones among Asian patients. Thus, the interaction of various risk factors for GSD may contribute to the different distribution of GSD between sexes in the Asian population compared with western populations $^{(3,4,13,16)}$. Studies elucidating the relationship between obesity and GSD in the Asian population are scarce. As the diet habits among Asians become westernised, there is increasing evidence demonstrating the impact of obesity on GSD risk in Asian people. However, few studies clarified the strength of the association between different obesity indicators and GSD risk.

Obesity is a major health issue because of its close association with many important chronic diseases, such as diabetes mellitus, CVD, etc. ${ }^{(17)}$. Obesity has long been recognised as an important risk factor for GSD, possibly due to hyper-saturated fat depositions releasing cytokines via the inflammatory cascade, leading to gallbladder dysfunction ${ }^{(9,15,18,19)}$. Many epidemiological studies demonstrated that obesity increases the risk of $\operatorname{GSD}^{(1,3-9)}$, but few of them clarified the difference between obesity indicators, such as BMI, WC and \%FM. BMI as an overall obesity parameter does not reflect adiposity related to abdominal obesity. Abdominal obesity has been shown to be closely related to the risk of GSD. Thus, we included other obesity indicators - WC and \%FM - to assess the risk of $\mathrm{GSD}^{(8,20,21)}$. Our results show that obesity, defined based on BMI, WC or \%FM, significantly increased the risk of GSD among women, even after adjusting for potential confounding factors. However, only BMI- and WC-defined obesity significantly increased the risk of GSD among men. The combination of all markedly increased obesity indicators was associated with a higher risk of GSD in both men and women. Among different obesity indicators, our study demonstrates that the best obesity indicator differed between men and women. Obesity, when defined based on WC, showed the strongest association with GSD, significantly greater than that \%FM-defined obesity, in men. In contrast, among women, obesity, when defined based on BMI, showed the strongest association with the presence of GSD. BMI is generally viewed as a parameter of general obesity, \%FM is considered to reflect the general adiposity of the body and WC is usually considered to estimate regional abdominal $\operatorname{adiposity}^{(8,9,20-22)}$. The weaker association between BMI and GSD in men than in women has been reported in previous studies and is considered to be due to the fact that men with a high BMI are more likely to have more lean body mass compared to women with high $\mathrm{BMI}^{(22)}$. As shown in Table 4 , we found that obesity, when defined by all obesity indicators, had a stronger relationship with the risk of GSD in women than in men. Few large studies demonstrated a different impact of obesity on health between men and women. Some studies showed that obesity-related metabolic syndrome better predicts early carotid atherosclerosis in women than in men ${ }^{(23,24)}$ However, further studies are needed to identify the different health effects of obesity according to sex.

There are some limitations to our study. First, it was crosssectional and we can only report the strength of the relationship between the obesity indicators and GSD risk. Prospective studies are needed to clarify the causal relationship between obesity indicators and GSD risk. Second, we used a lenient method to describe the presence of GSD. Further studies are needed to evaluate the possibility of a dose-response relationship between GSD severity, complications and each obesity indicator.

In conclusion, all obesity indicators predicted the risk of GSD in women but only BMI and WC were associated with GSD risk in men. A combination of all obesity indicators was related to the risk of GSD in both men and women. Obesity indicators are better predictors of the presence of GSD in women than in men. The best obesity indicator for GSD risk may differ by sex. In addition to body weight control, fat and central obesity are associated with the risk of GSD in women. However, in men, central obesity might play a more important role in the prediction of GSD risk.

\section{Acknowledgements}

The authors thank all members of the Department of Family Medicine, MacKay Memorial Hospital, Taipei, for help rendered for this study. Special thanks to Dr Lee-Ching Hwang who contributed to revising drafts of the manuscript. The authors also thank $\mathrm{Mr}$ Jing-Rong Jhuang for statistical technique consultation.

This research received no specific grant from any funding agency, commercial or not-for-profit sectors.

H.-Y. H., L.-C. H. participated in the design of the study, performed statistical analysis and interpretation of the data. H.-Y. H., L.-C. H. helped to draft the manuscript. H.-Y. H., C.-Y. H. and L.-C. H. contributed to revising drafts of the manuscript and all authors had the approval of the final manuscript.

The authors declare that there are no conflicts of interest.

\section{References}

1. Stinton LM, Myers RP \& Shaffer EA (2010) Epidemiology of gallstones. Gastroenterol Clin North Am 39, 157-169.

2. Stinton LM \& Shaffer EA (2012) Epidemiology of gallbladder disease: cholelithiasis and cancer. Gut Liver 6, 172-187.

3. Chen CH, Huang MH, Yang JC, et al. (2006) Prevalence and risk factors of gallstone disease in an adult population of Taiwan: an epidemiological survey. J Gastroenterol Hepatol Res 21, 1737-1743.

4. Zamani F, Sohrabi M, Alipour A, et al. (2014) Prevalence and risk factors of cholelithiasis in Amol city, northern Iran: a population based study. Arch Iran Med 17, 750-754.

5. Cruz-Monserrate Z, Conwell DL \& Krishna SG (2016) The impact of obesity on gallstone disease, acute pancreatitis, and pancreatic cancer. Gastroenterol Clin North Am 45, 625-637.

6. Camilleri M, Malhi H \& Acosta A (2017) Gastrointestinal complications of obesity. Gastroenterology 152, 1656-1670.

7. Premkumar M \& Sable T (2012) Obesity, dyslipidemia and cholesterol gallstone disease during one year of Antarctic residence. Rural Remote Health 12, 2186.

8. Tsai CJ, Leitzmann MF, Willett WC, et al. (2006) Central adiposity, regional fat distribution, and the risk of cholecystectomy in women. Gut 55, 708-714. 
9. Radmard AR, Merat S, Kooraki S, et al. (2015) Gallstone disease and obesity: a population-based study on abdominal fat distribution and gender differences. Ann Hepatol 14, 702-709.

10. Jensen KH \& Jorgensen T (1991) Incidence of gallstones in a Danish population. Gastroenterology 100, 790-794.

11. DeLong ER, DeLong DM \& Clarke-Pearson DL (1988) Comparing the areas under two or more correlated receiver operating characteristic curves: a nonparametric approach. Biometrics 44, 837-845.

12. Peltz G, Aguirre MT, Sanderson M, et al. (2010) The role of fat mass index in determining obesity. Am J Hum Biol 22, 639-647.

13. Zhu L, Aili A, Zhang C, et al. (2014) Prevalence of and risk factors for gallstones in Uighur and Han Chinese. World J Gastroenterol 20, 14942-14949.

14. You CH, Huang CY, Ow SP, et al. (2015) The relationship of serum lipid profiles and risk of gallstone disease in Taiwanese subjects. Taiwan J Fam Med 25, 129-137.

15. Lee YC, Wu JS, Yang YC, et al. (2014) Moderate to severe, but not mild, nonalcoholic fatty liver disease associated with increased risk of gallstone disease. Scand J Gastroenterol $\mathbf{4 9}$, 1001-1006.

16. Stringer MD, Fraser S, Gordon KC, et al. (2013) Gallstones in New Zealand: composition, risk factors and ethnic differences. ANZ J Surg 83, 575-580.
17. Guh DP, Zhang W, Bansback N, et al. (2009) The incidence of co-morbidities related to obesity and overweight: a systematic review and meta-analysis. BMC Public Health 9, 88.

18. Shabanzadeh DM, Skaaby T, Sorensen LT, et al. (2017) Metabolic biomarkers and gallstone disease - a populationbased study. Scand J Gastroenterol 52, 1270-1277.

19. Sodhi JS, Zargar SA, Khateeb S, et al. (2014) Prevalence of gallstone disease in patients with type 2 diabetes and the risk factors in North Indian population: a case-control study. Indian J Gastroenterol 33, 507-511.

20. Sekine K, Nagata N, Sakamoto K, et al. (2015) Abdominal visceral fat accumulation measured by computed tomography associated with an increased risk of gallstone disease. J Gastroenterol Hepatol Res 30, 1325-1331.

21. Aune D, Norat $\mathrm{T} \&$ \& Vatten LJ (2015) Body mass index, abdominal fatness and the risk of gallbladder disease. Eur J Epidemiol 30, 1009-1019.

22. Tsai CJ, Leitzmann MF, Willett WC, et al. (2004) Prospective study of abdominal adiposity and gallstone disease in US men. Am J Clin Nutr 80, 38-44.

23. Iglseder B, Cip P, Malaimare L, et al. (2005) The metabolic syndrome is a stronger risk factor for early carotid atherosclerosis in women than in men. Stroke 36, 1212-1217.

24. Kawamoto R, Tomita H, Inoue A, et al. (2007) Metabolic syndrome may be a risk factor for early carotid atherosclerosis in women but not in men. $J$ Atheroscler Thromb 14, 36-43. 